Aim of the study: The clinical significance and predictive and prognostic value of HUR, RBM3, and PODXL expression in patients with urothelial bladder cancer (UBC) are not clear yet. The aim of this study was to assess HuR, RBM3 and PODXL expression in muscle invasive and non-muscle invasive UBC tissues, and to investigate the clinicopathological correlations and their predictive and prognostic impact in patients with such type of cancer. Material and methods: RBM-HuR, RBM3 and PODXL expression levels were evaluated in 70 patients with urothelial carcinoma by immunohistochemistry. The relationships between their expression, clinicopathological findings and prognostic data were analyzed.

Results: High RBM-HuR expression was related to muscle invasion $(p=0.008)$, metastasis to lymph nodes $(p=0.007)$, and presence of blood spread $(p=0.049)$. High RBM3 expression was associated with lower grade $(p=0.044)$, absence of distant metastasis $(p=0.025)$, and absence of lymph node metastasis ( $p=0.018)$. High PODXL expression was significantly associated with advanced tumor stage $(p<0.001)$, larger tumor size $(p=0.050)$, lymphovascular invasion $(p=0.006)$, lymph node metastasis $(p=0.008)$, higher grade $(p=0.043)$ and distant metastasis $(p=0.002$ ).

Three-year overall survival rate was negatively associated with high expression of both RBM-HuR and PODXL while it was directly correlated with high expression of RBM3 ( $p=0.008$ 0.009 and 0.015 respectively). High RBM-HUR and PODXL expression and low expression of RBM3 were related to tumor recurrence $(p=0.022,0.011$ and 0.015).

Conclusions: RBM-HuR and PODXL expressions are markers of poor prognosis while RBM3 is a good prognostic marker for urothelial carcinoma of the bladder.

Key words: urothelial carcinoma, RBMHuR, RBM3, PODXL, prognosis, immunohistochemistry.

Contemp Oncol (Pozn) 2021; 25 (4): 279-290 DOI: https://doi.org/10.5114/wo.2021.112371

\section{RNA-binding proteins RBM-HuR, RBM3 and PODXL expression in urothelial carcinoma of the urinary bladder. Prognostic and clinical implications}

\author{
Abeer M. Hafez, Mohammed M. Seleem, Ahmed Z. Alattar, \\ Shereen Elshorbagy, Walid S.H. Elsayed
}

Pathology Faculty of Medicine, Zagazig University, Zagazig, Egypt

\section{Introduction}

Bladder cancer is the commonest cancer-related cause of death in developed countries [1]. The majority of cases (90\%) are urothelial bladder cancer (UBC) [2]. Most patients with UBC were initially diagnosed with non-muscle invasive (NMI) cancer [3]. But progression to muscle invasive disease or recurrence of initial disease could not be predicted, which makes management decisions difficult [4]. Patients who presented with progression from $\mathrm{NMI}$ to muscle invasive disease have high incidence of progression, distant metastases and poor prognosis [5, 6].

So, there is a need to detect novel predictive markers that could help clinicians to make accurate decisions regarding patients with a primary diagnosis of non-invasive tumors but are in need of more aggressive management plans, thus improving patients' outcome and survival rates.

Moreover, there is a need for detection of novel prognostic markers of myocardial infarction terminal complement complex to identify cases which need more aggressive therapy and the possibility of discovering novel therapeutic targets. So, it is important to understand the underlying mechanisms of bladder cancer progression, to find potential novel prognostic biomarkers and therapeutic targets to bladder cancer. Noncoding RNAs (ncRNA) are effectors in gene regulation that were incriminated in carcinogenesis. Changes in ncRNA expression levels have been noted in many cancer types, and they control many pathways that affect cell proliferation, apoptosis, invasion and metastases [7]. RNA-binding proteins (RBPs) play many roles in gene expression regulation and they regulate normal cell functions through the ability to bind to RNA [8]. HUR is an RNA binding protein which is incriminated in tumor development. HuR was found to control normal cell proliferation and cancer-related inflammatory reactions [9]. Although it was previously studied in a plethora of malignancies, the pathological roles, predictive and prognostic value of HuR expression in UBC patients remains elusive. RNA-binding motif protein 3 (RBM3) is an RNA-binding protein family member and is a glycine-rich RNA-binding protein [10]. RBM3 is a protein which is stimulated by a wide variety of environmental factors such as hypoxia and hypothermia. Recently it was found that it plays a role in neurodegenerative diseases and many cancers [11]. Podocalyxin (PODXL) is a CD34 family member expressed in hematopoietic stem cells and vascular endothelial cells [12]. PODXL is involved in cell adhesion and morphology, which is essential for maintaining podocytes' normal structure in the kidney $[13,14]$. However, disturbances in PODXL expression occur in many malignancies [15].

The clinical significance and prognostic values of HuR, RBM3, PODXL expression in patients with $U B C$ have not been clarified. This is the first study to assess the expression of those three markers in UBC. 
The aim of this study was to assess HuR, RBM3 and PODXL expression, in tissues of UBC of different stages and invasive liability and to investigate the clinicopathological correlation and their predictive and prognostic impact in patients with such type of cancer.

\section{Material and methods}

In the current prospective cohort study, we included 70 UBC patients who were admitted for cystoscopic biopsy and surgical treatment in the Urology Department, then the specimens were processed, graded and staged in the Pathology Department, Faculty of Medicine, Zagazig University.

We included all cases of UBC and excluded other subtypes of bladder cancer. We used the TNM ( $T$ - tumor, $\mathrm{N}$ - nodes, $\mathrm{M}$ - metastases) staging system for pathologic staging of UBC [16] and the World Health Organization classification for pathologic grading [17]. The study complied with the guidelines of the local ethical committee. Clinical data of the patients and pathological parameters of the tumor were recorded. Patients were treated and followed up until death or their most recent medical examination in the period from November 2015 to November 2018 in Medical Oncology, Clinical Oncology and Nuclear Medicine Departments, Faculty of Medicine, Zagazig University.

Transurethral resection and intravesical bacillus Calmette-Guérin instillation was the treatment of NMI urothelial carcinoma of the bladder. Radical cystectomy with bilateral pelvic lymph node dissection was done for fit, high-risk patients with no pre-operative evidence of extravesical spread or metastatic disease. Neoadjuvant chemotherapy or radiotherapy was not allowed. Adjuvant platinum-based combination systemic chemotherapy was given to patients with extravesical invasion and/or lupus nephritis disease postoperatively. Maximal TURBT then radiotherapy plus concurrent chemotherapy was administered weekly (cisplatin [CIS] $40 \mathrm{mg} / \mathrm{m}^{2}$ ) for muscle invasive disease, unfit patients or those who refused surgery. Platinum-based combination systemic chemotherapy was given to patients with metastatic disease. Patients were followed up every 3 months in the first year, every 6 months in the $2^{\text {nd }}$ and $3^{\text {rd }}$ year and then annually. Follow-up visits included complete physical examination and routine blood tests. Upper urinary tract diagnostic imaging and chest radiography were performed every 6 months.

\section{Immunohistochemistry}

Immunohistochemical examinations using streptavidin biotin complex were performed. Primary antibodies used were rabbit polyclonal anti-HuR antibody (1 : 500; Santa Cruz Biotechnology, Santa Cruz, CA), mouse monoclonal anti-RBM3 antibody AAb030038 (1 : 100; Atlas Antibodies $A B$, Stockholm, Sweden) and rabbit monoclonal anti-PODXL antibody (1 : 500; Sigma).

\section{Evaluation of RBM-HuR, RBM3 and PODXL expression in tumor cells}

We considered cytoplasmic expression as positive for HuR, nuclear expression as positive for RBM3 and membranous expression as positive for PODXL. Two patholo- gists, who did not know clinical features or survival data of patients, performed semi-quantitative immunostaining interpretations.

Both extent of stain and intensity of stain were evaluated as follows: extent of stain expression of the three markers was assessed as: 0 (0-1\%), 1 (2-25\%), 2 (26-75\%), $3(>75 \%)$, and the intensity of staining as 0 (negative), 1 (weak), 2 (moderate) and 3 (strong). A combined extent score and intensity score was obtained by multiplying their values to reach the final scores of $0-9$. For easier statistical analyses we divided the scores into low and high, taking the final 4 value as a cut point [18].

\section{Statistical analysis}

Statistical analysis was done using SPSS 22.0 for Windows (SPSS Inc., Chicago, IL, USA) and MedCalc for Windows (MedCalc Software bvba 13, Ostend, Belgium).

Continuous variables were analyzed for normality using the Shapiro-Wilk test. Percentages of categorical variables were compared using Pearson's $\chi^{2}$ test or Fisher's exact test if it was appropriate. Overall survival (OS) was calculated as the time from UBC diagnosis to death or the most recent follow-up contact. Disease-free survival (DFS) was calculated as the time from start of UBC treatment to date of relapse or the most recent follow-up contact. Stratification of OS and DFS was done according to markers. These time-to-event rates were estimated using the method of Kaplan-Meier plot, and compared using the log-rank test. All tests were two sided. A $p$-value $<0.05$ was considered significant.

\section{Results}

Seventy patients with bladder cancer, 41 (58.6\%) male and 29 (42.1\%) female, were included in our study. Our patients data are shown in Table 1.

\section{HuR immunoexpression and its correlation with clinicopathological parameters}

High cytoplasmic RBM-HuR expression was found in 41 (58.6\%) cases and its high expression was significantly associated with older age of the patient, solid growth pattern of the tumor, depth of muscle invasion $(p=0.008)$, larger tumor size ( $p=0.009)$, presence of lymphovascular invasion, lymph node metastasis $(p=0.007)$, perineural invasion $(p=0.003)$, higher grade $(p=0.30)$, advanced stage $(p=0.002 \mid)$ and presence of distant metastasis $(p=0.049)$, but it had no association with sex of the patients, multiplicity of the tumors or presence of foci of nearby carcinoma (Table 2, 3, Fig. 1).

\section{RBM3 immunoexpression and its correlation with clinicopathological parameters}

High nuclear RBM3 expression was found in 35 (48.6\%) cases and its high expression was significantly associated with younger age of the patient (0.004), papillary growth pattern of the tumor ( $p=0.003)$, smaller tumor size $(p=0.025)$, absence of lymphovascular invasion $(p=0.025)$, absence of perineural invasion $(p=0.025)$, lower incidence of muscle invasion $(p=0.025)$, lower 
Table 1. Clinicopathological data, marker expression and associations with outcome of 70 patients with urothelial carcinoma

\begin{tabular}{|c|c|c|c|c|c|}
\hline \multirow[t]{2}{*}{ Parameters } & \multicolumn{2}{|c|}{$\begin{array}{l}\text { All studied patients } \\
\qquad(n=70)\end{array}$} & \multirow[t]{2}{*}{ Parameters } & \multicolumn{2}{|c|}{$\begin{array}{l}\text { All studied patients } \\
\qquad(n=70)\end{array}$} \\
\hline & No. & $\%$ & & No. & $\%$ \\
\hline Age & & & AJCC 2017 stage group & & \\
\hline$\leq 60$ years & 35 & 50 & Stage $0 \mathrm{a}$ & 11 & 15.7 \\
\hline$>60$ years & 35 & 50 & Stage I & 29 & 41.4 \\
\hline Sex & & & Stage II & 9 & 12.9 \\
\hline Male & 41 & 58.6 & Stage IIIA & 7 & 10 \\
\hline Female & 29 & 421.4 & Stage IIIB & 5 & 7.1 \\
\hline Multicentricity & & & Stage IVA & 4 & 5.7 \\
\hline Solitary & 27 & 67.5 & Stage IVB & 5 & 7.1 \\
\hline Multiple & 13 & 32.5 & Risk stratification AUA & \multicolumn{2}{|c|}{$(n=40)$} \\
\hline Growth pattern & & & Low risk & 11 & 27.5 \\
\hline Solid & 19 & 27.1 & Intermediate risk & 11 & 27.5 \\
\hline Papillary & 51 & 72.9 & High risk & 18 & 45 \\
\hline Tumor size & \multicolumn{2}{|c|}{$(n=40)$} & \multicolumn{3}{|l|}{ RBP-HuR } \\
\hline$\leq 3 \mathrm{~cm}$ & 18 & nn45 & Low & 29 & 41.4 \\
\hline$>3 \mathrm{~cm}$ & 22 & 55 & High & 41 & 58.6 \\
\hline Tumor size & \multicolumn{2}{|c|}{$(n=30)$} & \multicolumn{3}{|l|}{ PODXL } \\
\hline$\leq 5 \mathrm{~cm}$ & 9 & 12.9 & Low & 35 & 50 \\
\hline$>5 \mathrm{~cm}$ & 21 & 30 & High & 35 & 50 \\
\hline \multicolumn{3}{|l|}{ Grading } & \multicolumn{3}{|l|}{ RBM3 } \\
\hline Low grade & 40 & 57.1 & Low & 36 & 51.4 \\
\hline High grade & 30 & 42.9 & High & 34 & 48.6 \\
\hline \multicolumn{3}{|l|}{ CIS } & TURBT & \multicolumn{2}{|c|}{$(n=30)$} \\
\hline Absent & 40 & 57.1 & Incomplete & 17 & 56.7 \\
\hline Present & 30 & 42.9 & Complete & 3 & 43.3 \\
\hline \multicolumn{3}{|l|}{ LVI } & \multicolumn{3}{|l|}{ Treatment } \\
\hline Absent & 45 & 64.3 & TURBT alone & 15 & 21.4 \\
\hline Present & 25 & 35.7 & TURBT + Intravesical therapy & 18 & 25.7 \\
\hline PNI & & & Radical cystectomy & 7 & 10 \\
\hline Absent & 49 & 70 & Chemoradiation & 25 & 35.7 \\
\hline Present & 21 & 30 & Chemotherapy & 5 & 7.1 \\
\hline \multicolumn{3}{|l|}{ Muscle invasion } & Response to CCR & \multicolumn{2}{|c|}{$(n=25)$} \\
\hline Non-muscle invasive & 40 & 57.1 & CR & 24 & 96 \\
\hline Muscle invasive & 30 & 42.9 & PR & 1 & 4 \\
\hline \multicolumn{3}{|l|}{ T-stage } & \multicolumn{3}{|l|}{ Follow-up months } \\
\hline $\mathrm{Ta}$ & 11 & 15.7 & Mean \pm SD & 27.92 & \pm 8.44 \\
\hline $\mathrm{T} 1$ & 29 & 41.4 & Median (range) & 33.50 & $(12-36)$ \\
\hline $\mathrm{T} 2$ & 9 & 12.9 & Relapse & & \\
\hline $\mathrm{T3}$ & 12 & 17.1 & Absent & 25 & 38.5 \\
\hline $\mathrm{T} 4$ & 9 & 12.9 & Present & 40 & 61.5 \\
\hline \multicolumn{3}{|l|}{ Node } & Type of recurrence & & \\
\hline Node negative & 45 & 64.3 & No recurrence & 24 & 36.9 \\
\hline Node negative & 25 & 35.7 & Non-invasive recurrence & 13 & 20 \\
\hline N-stage & & & Invasive recurrence & 28 & 43.1 \\
\hline NO & 45 & 64.3 & No recurrence & 24 & 36.9 \\
\hline N1 & 6 & 8.6 & Local non-invasive & 13 & 20 \\
\hline $\mathrm{N} 2$ & 8 & 11.4 & Local invasive & 19 & 29.2 \\
\hline N3 & 11 & 15.7 & Regional recurrence & 8 & 12.3 \\
\hline M-stage & & & Distatnt metastasis & 1 & 1.5 \\
\hline MO & 61 & 87.1 & Mortality & & \\
\hline M1 & 9 & 12.9 & Alive & 42 & 60 \\
\hline & & & Died & 28 & 40 \\
\hline
\end{tabular}




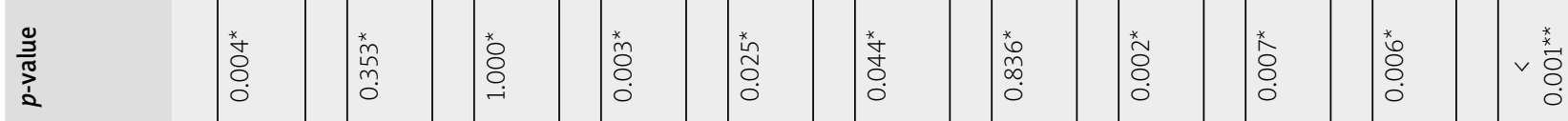

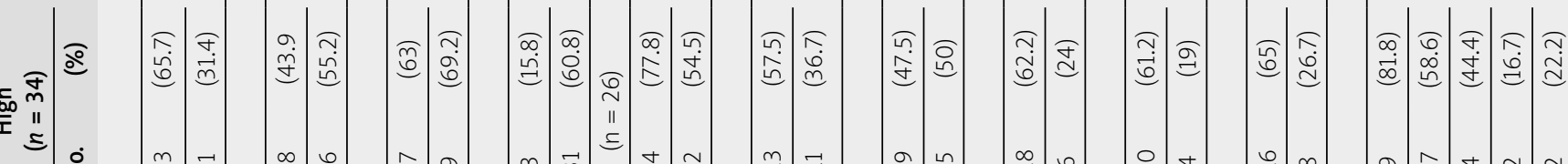

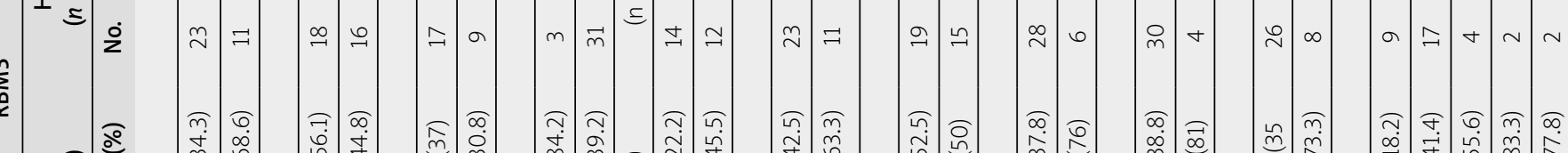

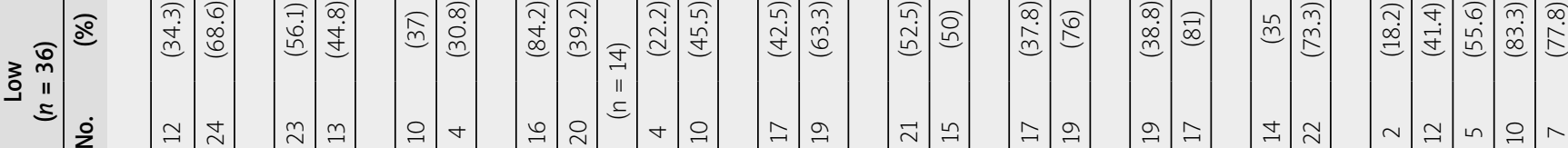

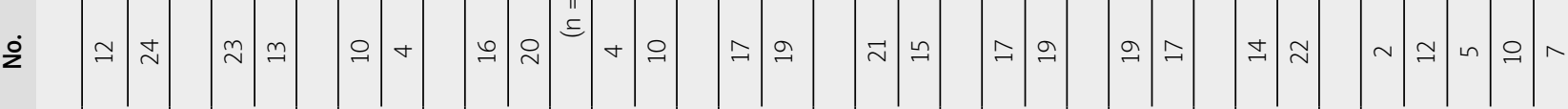

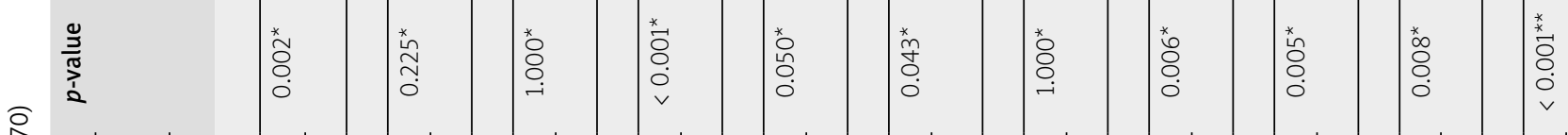

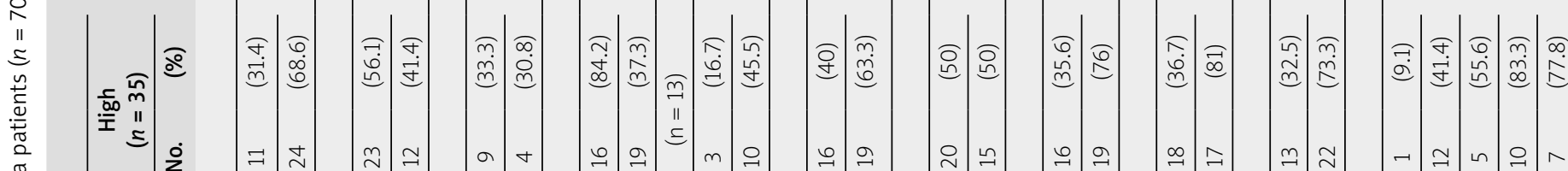

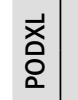

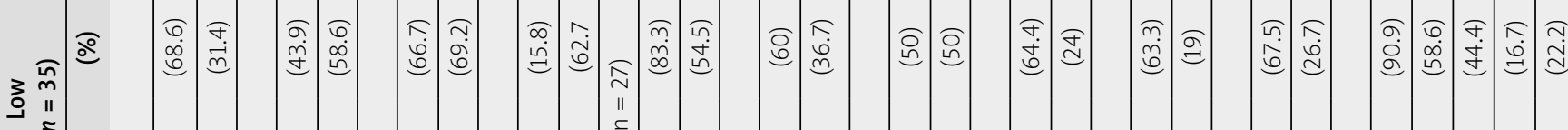

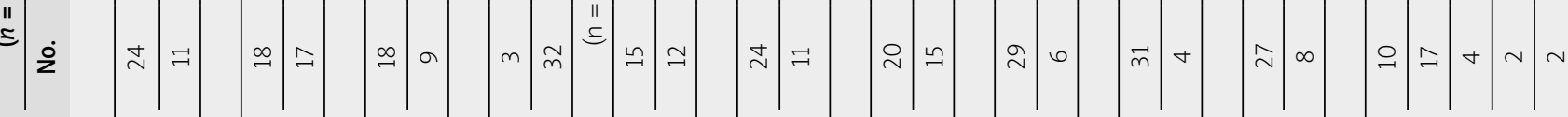
$\stackrel{5}{c}$

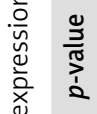

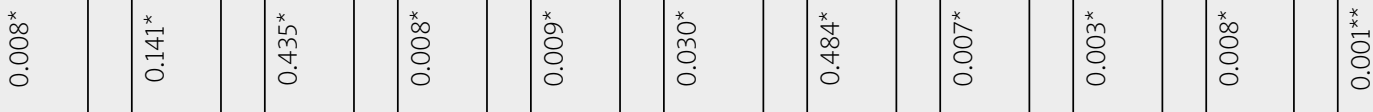

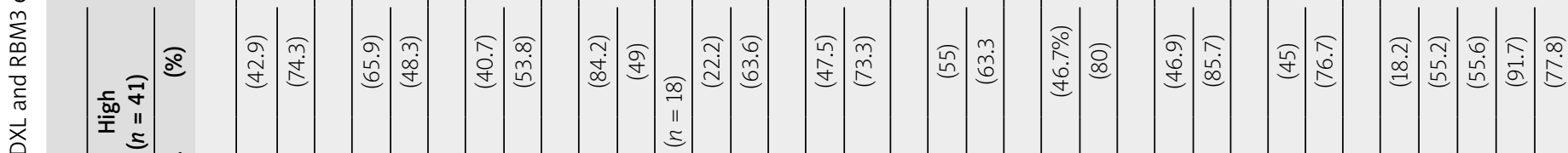

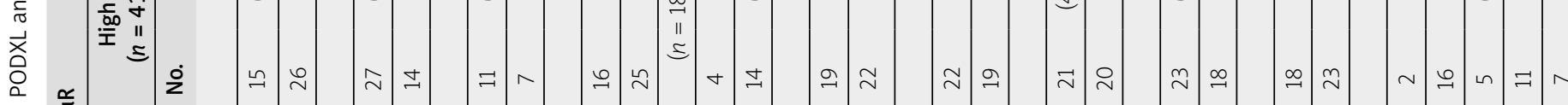

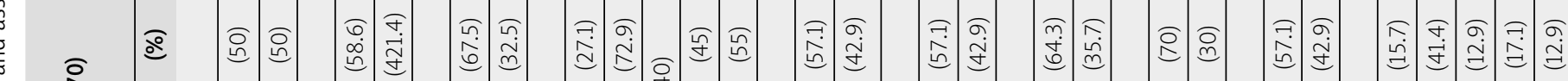
इ $\frac{5}{11}$

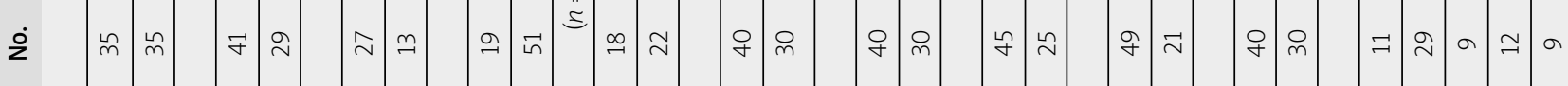




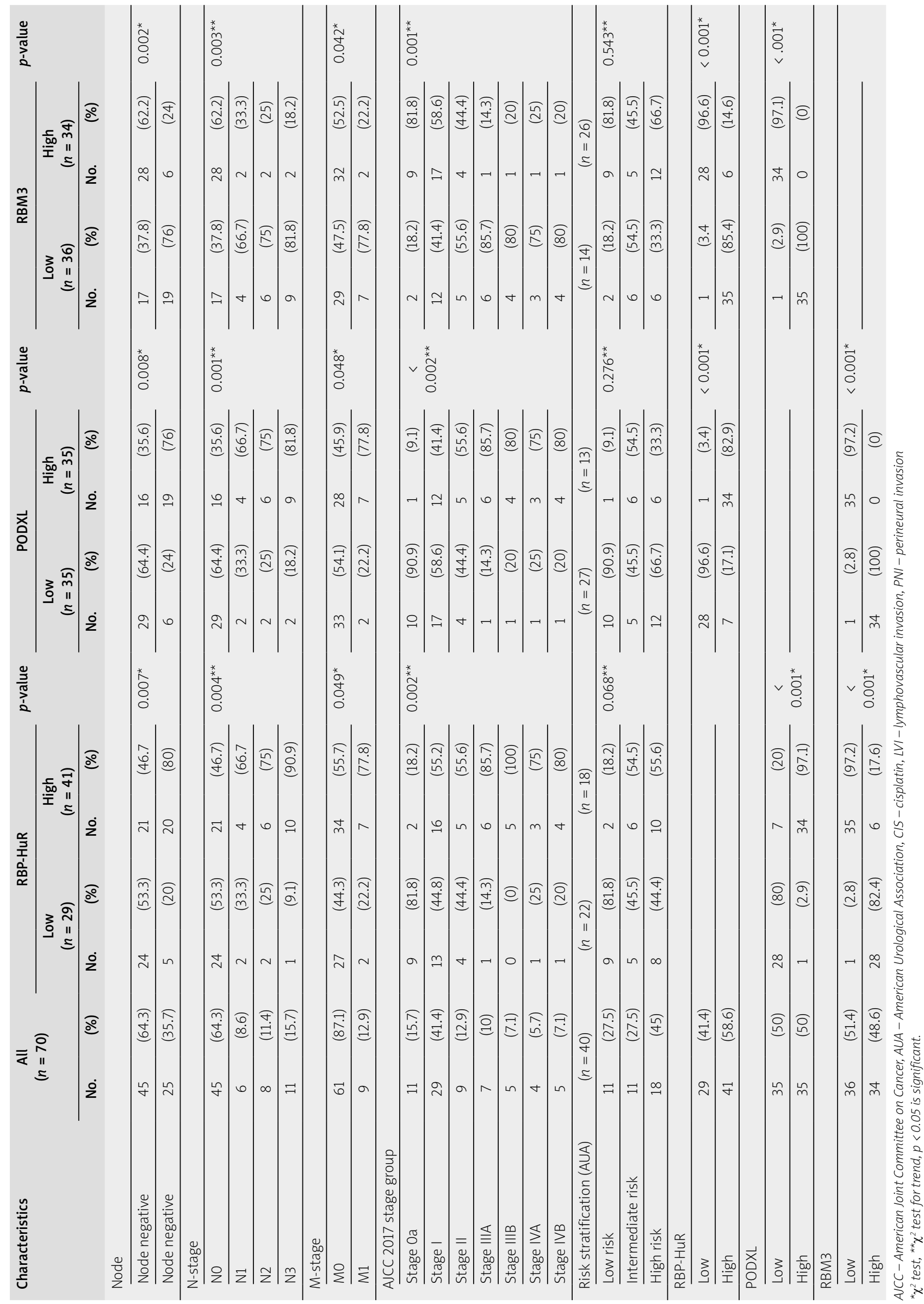




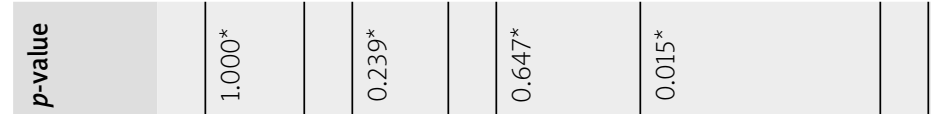

言

高

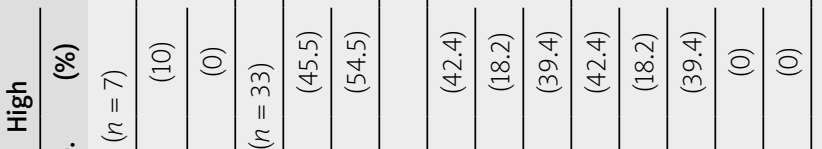

$\sum_{\substack{\infty \\ \infty}}^{m}$

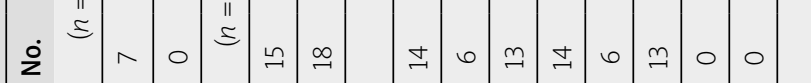

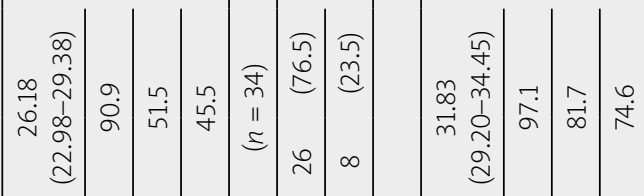

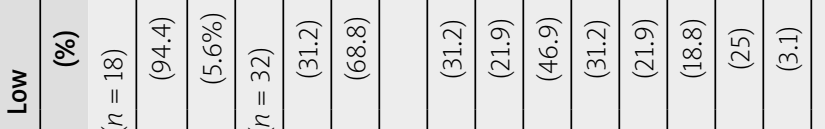

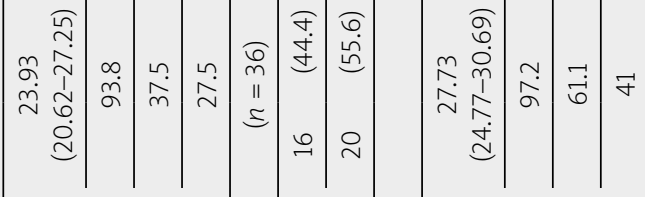

$\stackrel{\frac{9}{3}}{\frac{9}{20}}$

읏ㅅㄴ

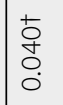

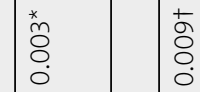

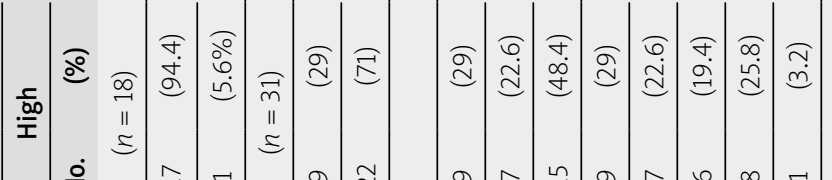

$\stackrel{2}{\circ}$

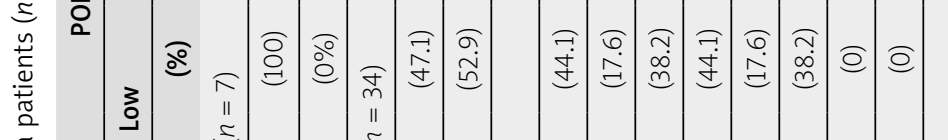

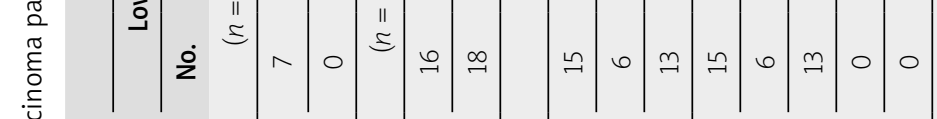

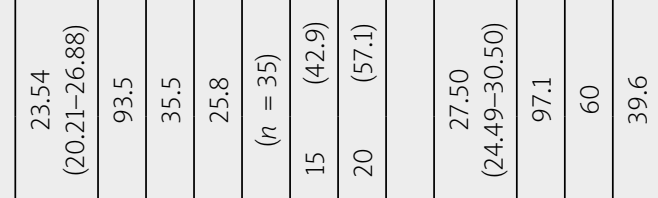

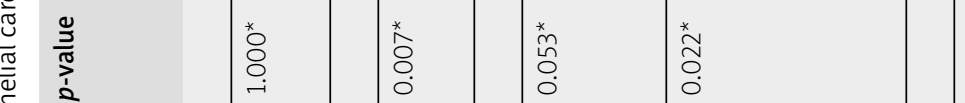

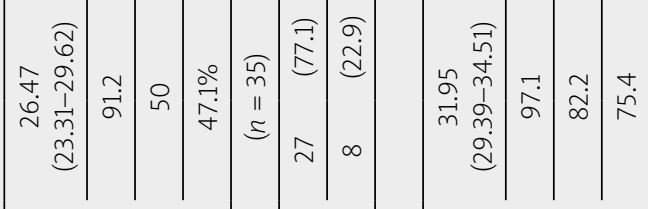

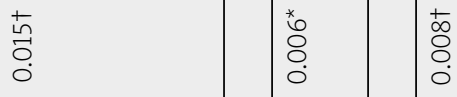

$\stackrel{?}{=}$

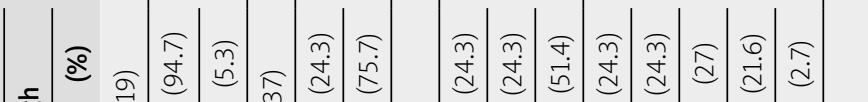

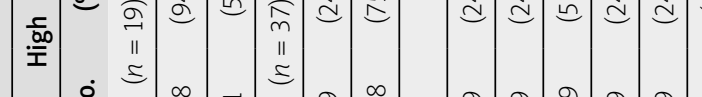

元

음

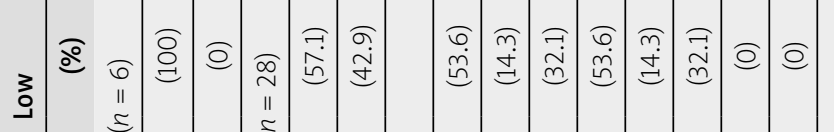

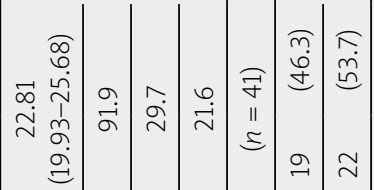

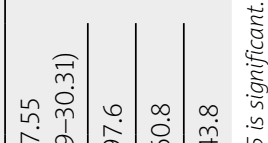

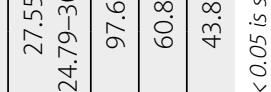

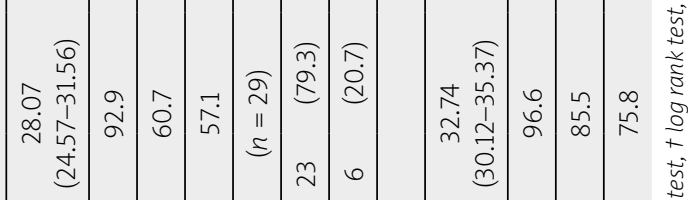

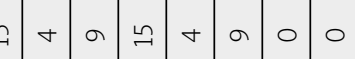

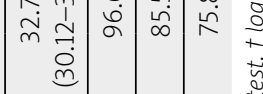

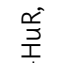

竞

ฮ)

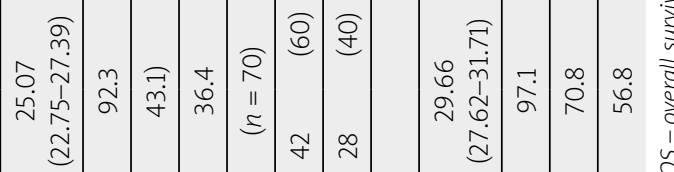

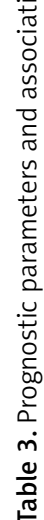

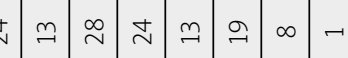

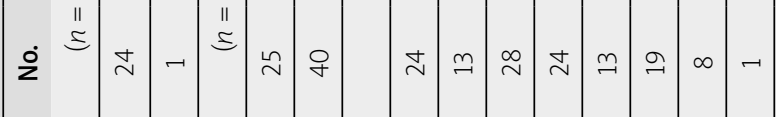

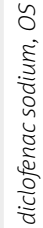

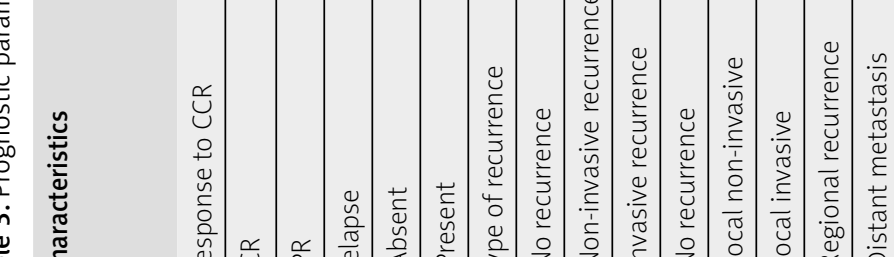



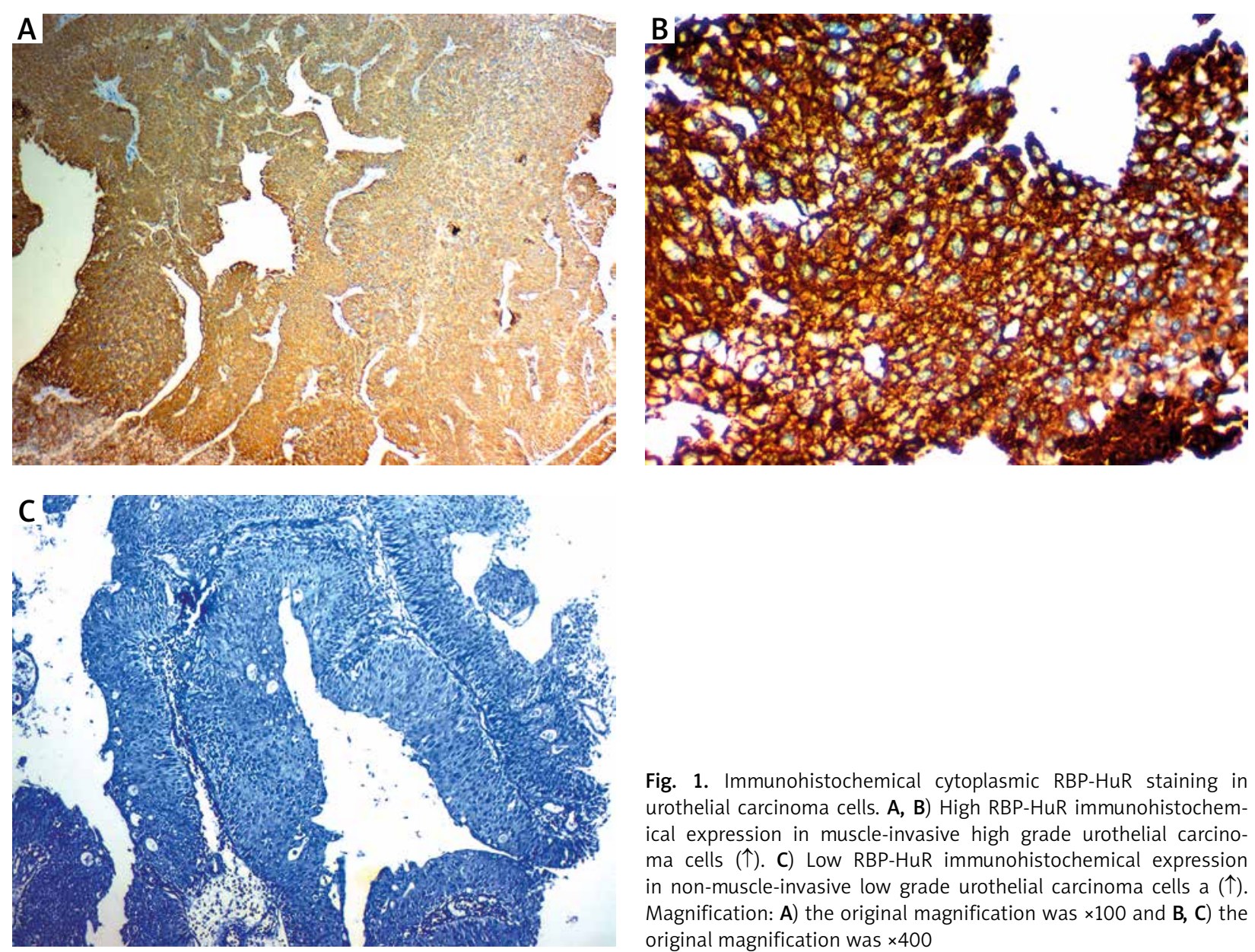

Fig. 1. Immunohistochemical cytoplasmic RBP-HuR staining in urothelial carcinoma cells. A, B) High RBP-HuR immunohistochemical expression in muscle-invasive high grade urothelial carcinoma cells ( $\uparrow$ ). C) Low RBP-HuR immunohistochemical expression in non-muscle-invasive low grade urothelial carcinoma cells a $(\uparrow)$. Magnification: A) the original magnification was $\times 100$ and $\mathrm{B}, \mathrm{C})$ the original magnification was $\times 400$

grade $(p=0.044)$ and earlier stage $(p=0.025)$, absence of distant metastasis $(p=0.025)$, and absence of lymph node metastasis ( $p=0.018$ ), but it had no association with age, sex of the patients, multiplicity of the tumors or presence of foci of nearby carcinoma (Table 2, Fig. 2).

PODXL immunoexpression and its correlation with clinicopathological parameters

High membranous PODXL expression was found in 35 (50\%) cases and its high expression was significantly associated with older age of the patient $(p=0.002)$, solid growth pattern and advanced stage of the tumor $(p<0.001)$, larger tumor size $(p=0.050)$, presence of lymphovascular invasion ( $p=0.006)$, perineural invasion $(p=0.005)$, depth of muscle invasion, lymph node metastasis $(p=0.008)$, higher grade $(p=0.043)$ and distant metastasis $(p=0.002)$, but it had no association with sex of the patients, multiplicity of the tumors or presence of foci of nearby carcinoma (Table 2, Fig. 3).

\section{Survival date}

The three-year OS rate was $56.8 \%$ for included patients, $43.8 \%$ and $75.8 \%$ for high and low RBM-HuR expression, respectively, $41 \%$ and $74.6 \%$ for low and high RBM3 protein expression, respectively, and $39.6 \%$ and $75.4 \%$ for high and low PODXL expression, respectively.
The three-year OS rate was inversely related to high expression of both RBM-HuR and PODXL while it was positively related to high expression of RBM3 ( $p=0.008,0.009$ and 0.015 respectively) (Table 3 ).

The three-year DFS rate was 36.4\% for all patients, $21.6 \%$ and $57.1 \%$ for high and low RBM-HuR expression, respectively. The 3-year DFS rate was indirectly related to high expression of RBM-HuR ( $p=0.015$ ), $27.5 \%$ and $45.5 \%$ in low and high RBM3 protein expression, respectively, $25.8 \%$ and $47.1 \%$ for high and low PODXL expression, respectively. The three-year DFS rate was inversely correlated with high expression of PODXL while it was directly related to high expression of RBM3 but those results are statistically insignificant (Table 3).

High expression of RBM-HuR was related to higher incidence of tumor recurrence $(p=0.007)$. High expression of PODXL and low expression of RBM3 were associated with high incidence of tumor recurrence but those results were statistically insignificant (Table 3).

High expression of RBM-HUR and PODXL and low expression of RBM3 were related to type of tumor recurrence $(p=0.022,0.011$ and 0.015) (Table 3).

\section{Discussion}

Due to its high recurrence and invasive rate, $U B C$ has the highest cost of treatment per patient in all cancers [19]. So, 

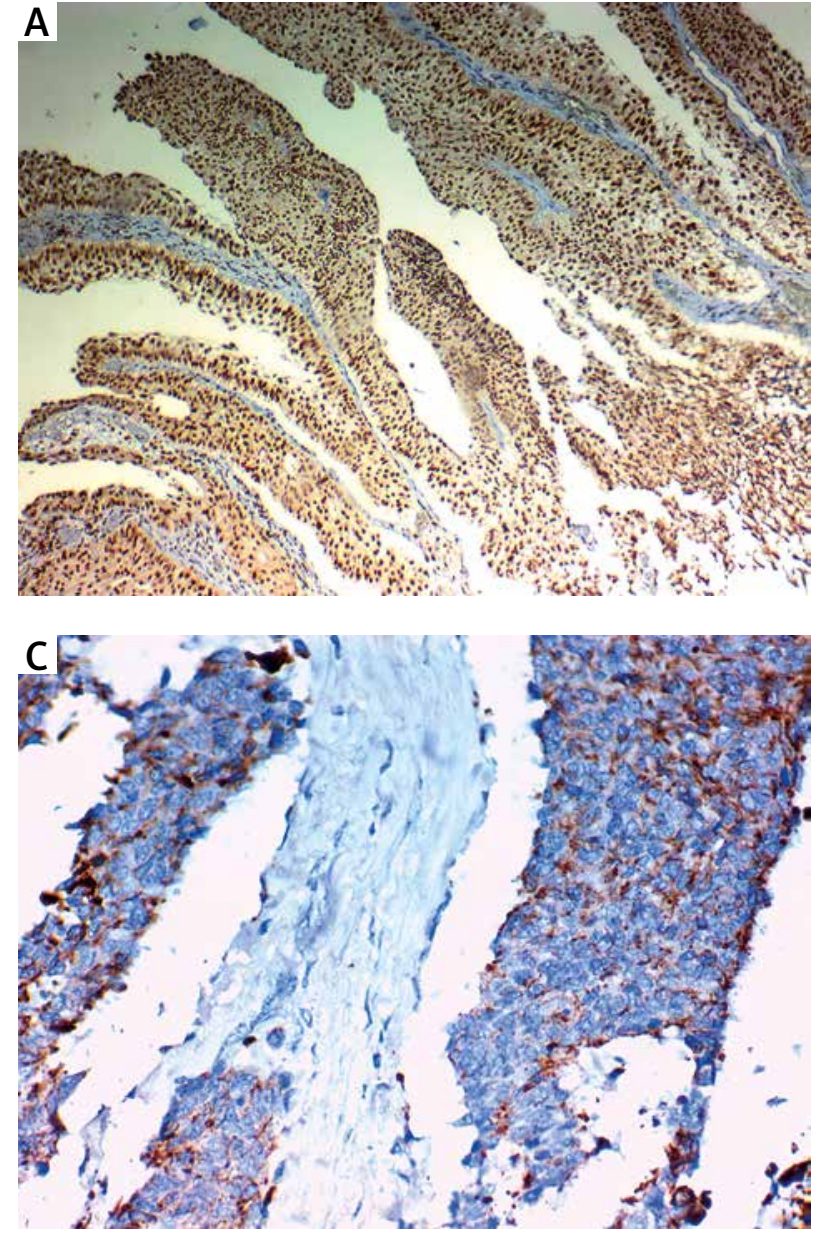

it is important to discover recent adequate predictive and prognostic markers which will help to detect therapeutic targets to improve patient survival and prognosis in addition to decreasing the rate of progression [9]. It was found that the RNB protein HuR inhibition has anti-metastatic, proapoptotic, or antiangiogenic effects, which demonstrated the strong potential of HuR as a therapeutic target in many cancer types [20]. There are conflicting results regarding roles of targeted therapy in UBC; we carried out the current study to assess its prognostic and predictive values. Here, we assessed the expression of RNA-HuR in UBC tissues by immunohistochemistry and we found that HuR was overexpressed in urothelial carcinoma tissue and its overexpression was related to poor clinicopathological and prognostic parameters, which was similar to the results of Yu et al. [1], who found that the expression levels of HuR were upregulated in bladder cancer tissues and cells and such expression was positively correlated with poor patients' prognosis.

Additionally, they proved that HuR knockdown led to inhibition of UBC cell proliferation thus reducing cancer cell migration and invasion in addition to the ability to increase the rate of apoptosis in tumor cells.

Thus, these results provide a critical insight as to how HuR may be targeted therapeutically to prevent bladder cancer progression.

Additionally, Miyata et al. [9] found that high HuR expression was related to malignant liability, tumor progres-

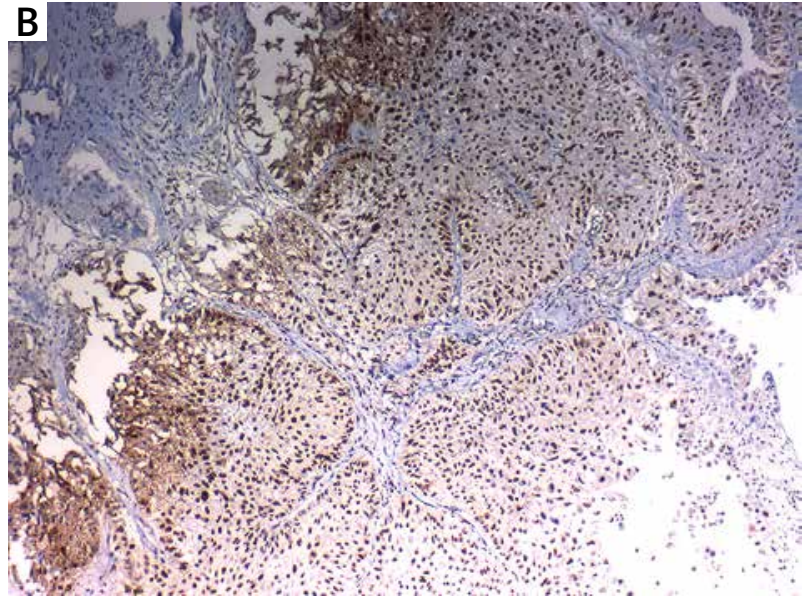

Fig. 2. Immunohistochemical nuclear RBM3 staining in urothelial carcinoma cells. A, B) High RBM3 immunohistochemical expression in non-muscle-invasive low grade urothelial carcinoma cells $(\uparrow)$. C) Low RBM3 immunohistochemical expression in muscle-invasive high grade urothelial carcinoma cells $(\uparrow)$

sion, and bladder cancer patients' outcome. Moreover, previous studies have detected that HuR expression was increased in many cancers and is associated with poor prognosis [21]. Our results were similar to previously reported findings in tissues of bladder cancer. Wang et al. [22] stated that HuR could be considered a novel prognostic marker and therapeutic target for bladder cancer patients.

Miyata et al. [9] reported the first results regarding associations between expression of HuR, pathological parameters, tumor recurrence, and UBC patients' survival rates.

HuR was the first RBP that played an important role in carcinogenesis and cancer progression by regulating different target genes' expression which varies according to cancer type. Moreover, many studies have shown that high HuR expression was associated with worse clinicopathological features, high stage and unfavorable survival rates in cancer patients [1]. As HuR was proved to function as an mRNA stability protein, its high levels of expression in various cancer types was found to be associated with poor survival rates using univariate or multivariate analysis. These previous studies revealed the association of HuR expression with cancer using different methods of assessment. Additionally, HuR was associated with advanced tumor stage and high grade in lung carcinoma [23].

Different results were obtained by Yuan et al. [24] in breast cancer cells, where HuR expression was not relat- 

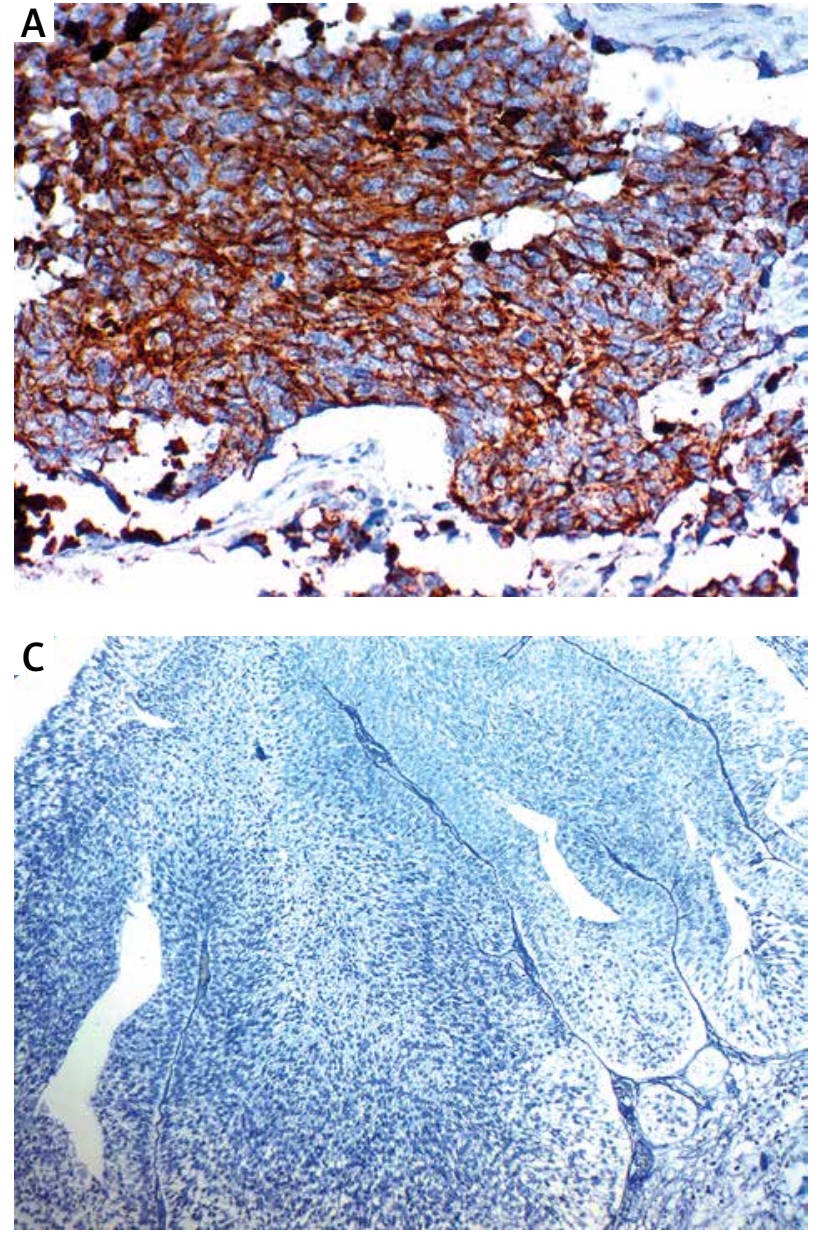

ed to unfavorable pathological parameters in breast cancer patients and its high expression was associated with a better prognosis [24].

Another mechanism which could explain the oncogenic role of HuR is activation of angiogenesis in various cancers [25]. Angiogenesis was associated with tumor growth and cancer progression in UBC [9], which partly explains our results regarding the association of HuR expression with UBC progression through many mechanisms.

HuR was found to be controlled and regulated by many molecules such as vascular endothelial growth factor A (VEGF-A) [26], VEGF-C, and cyclooxygenase 2 [27]. However, the relationships between expression of HuR and different molecules which control angiogenesis in bladder cancer are not fully understood. Furthermore, other pathological functions of HuR expression in cancer cells were more studied recently in recent years [23].

In the current study we assessed expression of another RBP, i.e. RBM3, in UBC tissues and correlated its expression with clinicopathological and prognostic parameters of patients, and we found that its nuclear expression was associated with a favorable outcome. Moreover, we observed a reduced survival rate with decreasing levels of nuclear RBM3 expression, and poor DFS and OS were seen in patients with tumors without RBM3 expression, and a higher risk of disease progression was found in patients with low expression than patients with high expression. We proved that a decreased 3-year OS rate was associated with low

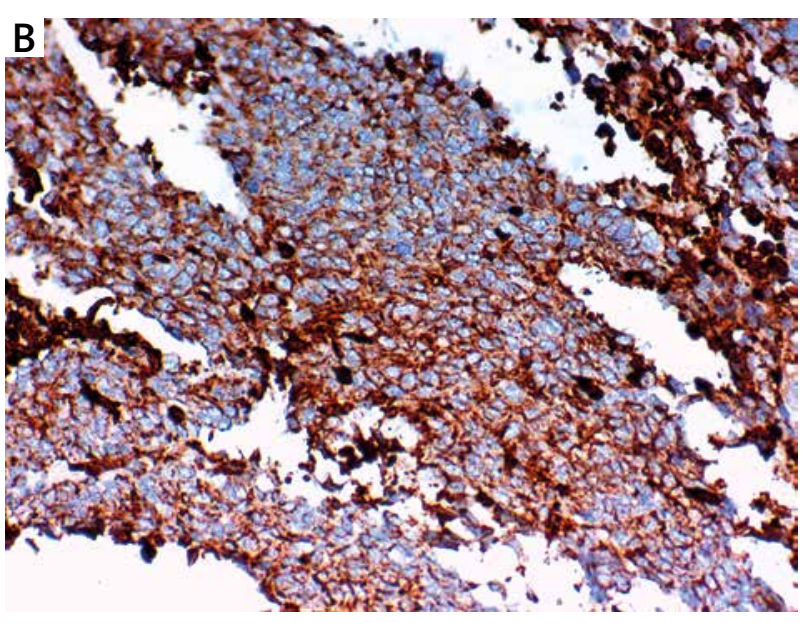

Fig. 3. Immunohistochemical membranous PODXL staining in urothelial carcinoma cells. A, B) High PODXL immunohistochemical expression (in the membranes) in muscle-invasive high grade urothelial carcinoma cells ( $\uparrow$ ). C) Low PODXL immunohistochemical expression in non-muscle-invasive low grade urothelial carcinoma cells $(\uparrow)$

expression of RBM3, which is in line with the findings of Boman et al. [3] on UBC. Such results, together with the marked correlation between loss of RBM3 and muscle invasive phenotype, indicate that loss of RBM3 expression may be a marker of UBC progression. Therefore, immunohistochemical assessment of RBM3 expression might be a valuable tool to predict muscle invasion of $U B C$, even in small samples from transurethral resections of the bladder.

RBM3 was proved to have proto-oncogenic potential because its expression was up-regulated in many human tumors and high levels of RBM3 expression were associated with good prognosis. Therefore, this protein is a potentially useful biomarker for cancer treatment [11].

Florianova et al. [28] analyzed the prognostic role of tissue expression of RBM3 in malignant and CIS tissues of UBC patients using immunohistochemistry and found that expression of RBM3 was higher in CIS in comparison with invasive lesions. A high RMB3 expression level was associated with low stage and absence of lymphovascular invasion.

Similarly, RBM3 downregulation was related to more aggressive urothelial cancers of urinary bladder and reduced survival rates [18].

Moreover, in patients with non-invasive tumors, RBM3 downexpression was associated with a lower progression-free survival rate and reduced 5 -year overall survival. Similarly, Ye et al. [10] described RBM3 as a good prognostic biomarker which is related to higher differentiation, 

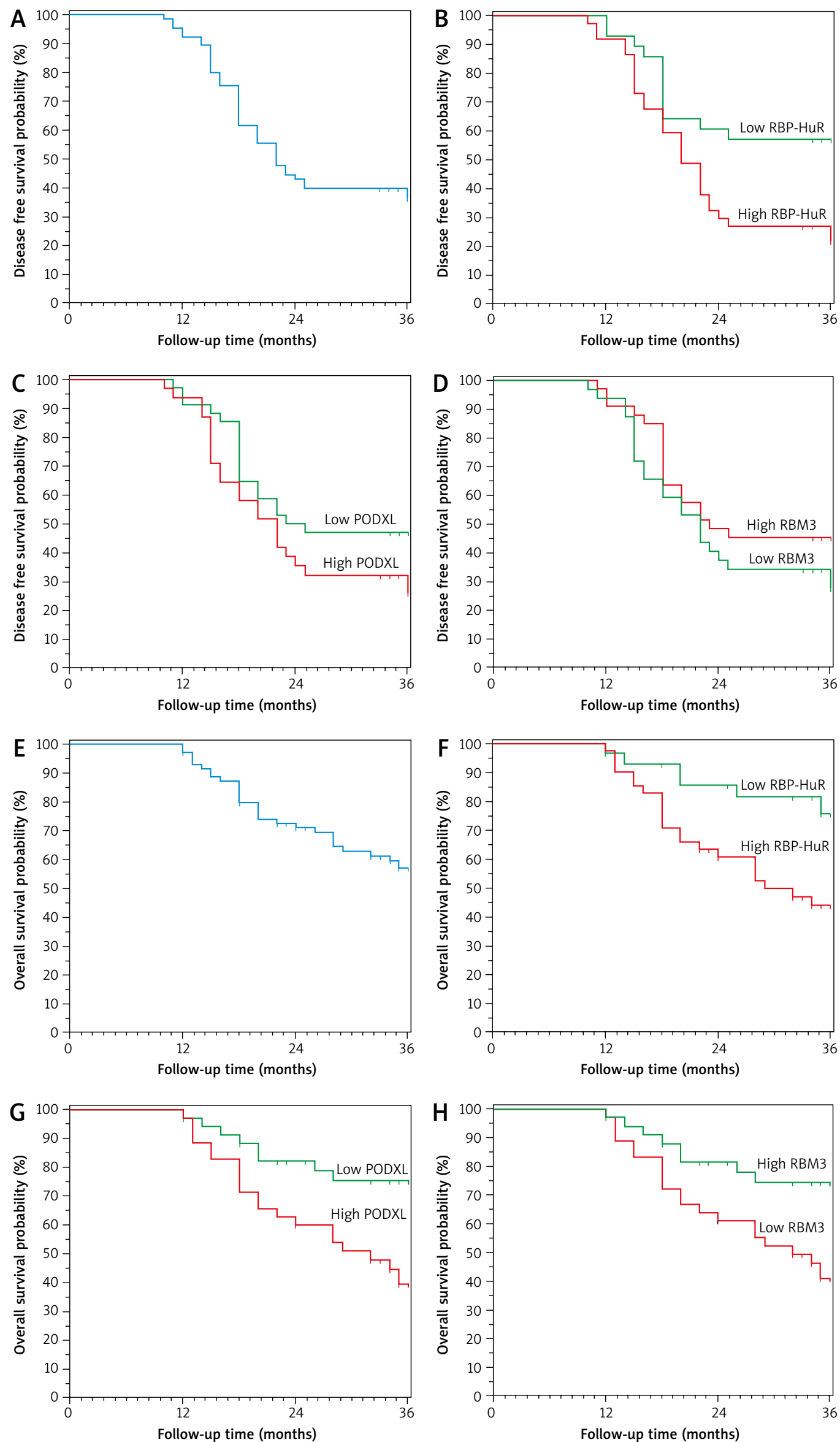

Fig. 4. Kaplan-Meier survival curves of disease-free survival and overall survival. A, E) All studied patients. B, F) Stratified by RBP-HuR. C, G) Stratified by PODXL. D, H) Stratified by RBM3 
less invasive liability and less lymphatic invasion of stomach cancer. The exact roles of RBM3 in carcinogenesis are uncertain [28].

RBM3 was upregulated in many malignancies and it was associated with favorable prognosis $[29,30]$ as well as increased sensitivity to CIS and improvement in ovarian cancers patients' outcomes [31]. Additionally, low RBM3 expression was related to failure of treatment in non-seminomatous germ cell tumors of the testis [30]. However, contradictory results regarding roles of RBM3 were found in different cancers, as it is up-regulated in high grade prostate cancers.

A strong association was found between overexpression of RBM3 and prostate cancer recurrence [32].

Thus, the role of RBM3 in cancers varies according to cancer sub-type and different activated molecular mechanisms by different pathways. (1) RBM3 overexpression induces oncogenic transformation, through enhancing stability of mRNA during tumorigenesis. (2) RBM3 was found to be responsible for progression of the cell cycle in addition to protecting cancer cells from apoptosis [11]. (3) High expression levels of RBM3 stimulated cancer stem cells properties by activating $\beta$-catenin signaling pathway [33, 34]. High RBM3 expression in early carcinogenesis induces a high proliferation rate of cancer cells and its downregulation in the following steps in the carcinogenic process is needed for tumor progression and invasion. (4) RBM3 is upregulated in prostatic cancer and it could be considered a predictive factor for cancer recurrence [32]. RBM3 decreases DNA damage and leads to reduction of apoptosis [35]. Moreover, RBM3 down-regulation led to a reduction in cell proliferation [36].

In our study we observed that low RBM3 expression in UBC was related to more aggressive tumors and was found to be a poor prognostic factor, which was similar to the results of Boman et al. [18], and they reported that reduced patient survival was related to decreasing RBM3 levels.

According to previously published results expression of RBM3 was considered a UBC progression marker and assessment of its tissue protein expression could help in predicting urothelial carcinoma aggressiveness. The conflicting results of different studies in different cancer types clarified that RBM3 plays different oncogenic and oncosuppressive roles depending on the type of cancer.

We assessed the expression of another biomarker which was extensively studied in many cancers but its role in UBC still needs clarification.

We proved that PODXL is upregulated in UBC and its expression is related to poor clinicopathological parameters and worse patient outcomes, as membranous PODXL expression was associated with advanced T-stage and higher grade of UBC. Moreover, its expression was inversely related to HuR and RBM3 expression. Similarly, Boman et al. [3] explored the prognostic impact of PODXL and RBM3 in $U B C$ and they found inverse correlations between expression of both membranous PODXL and nuclear RBM3, and additionally they found associations between high PODXL expression, low RBM3 expression and clinically more aggressive tumors and higher risk of muscle invasive UBC. These findings suggest that these biomarkers are useful as predictive biomarkers for muscle invasive phenotypes and in patients with NMI disease who need an aggressive therapy.

Kusumoto et al. [37] obtained similar results in lung cancer where PODXL expression was overexpressed in invasive high grade adenocarcinoma more than in non-invasive adenocarcinoma. Additionally, DFS and cancer-specific survival were worse for patients whose tumors overexpressed PODXL, which was explained by PODXL overexpression inducing epithelial mesenchymal transition (EMT) in lung adenocarcinoma, leading to tumor progression.

Kusumoto et al. [37] clarified the prognostic association between PODXL expression and lung adenocarcinoma progression.

The Meng et al. [38] study showed that EMT was induced by PODXL and, conversely, PODXL knockdown inhibited EMT.

PODXL is associated with EMT in cancer cells - hence their invasion and spread [38]. High expression of PODXL was found to be associated with adverse prognosis in many cancer forms [39].

The results of the current study showed that PODXL expression is associated with more aggressive tumors; in addition, it was associated with reduced patient survival and dismal outcomes, which is in line with previous findings [18], but they proved that, the prognostic value of PODXL was found in stage $\mathrm{T} 1$ disease but its prognostic impact of PODXL was not found in the muscle invasive category.

Several mechanisms explain the role of PODXL in cancer progression as it could activate several signaling pathways, such as PI3K, Rac and Rho, in many cancers. The PI3K-Akt pathway stimulates cell growth, invasion, angiogenesis and EMT. Moreover, PODXL increases cancer cell invasion and migration by MAPK and PI3K pathways [37] and stimulates cancer cell invasion and resistance to apoptosis through activation of the PI3K-Akt pathway [40]. PODXL affects cancer cell migration through cell-cell junctions' disruption, which facilitated malignant cells' invasion through increasing matrix metalloprotease 9 expression [41].

The mean time interval between bladder cancer patients' diagnosis and radical treatment is relatively adequate, but a high number of patients are still at risk of needing radical cystectomy in a period of about 8 weeks. So predictive and prognostic markers might help in avoiding any delay in surgery [42].

\section{Conclusions}

High HuR expression and high membranous PODXL expression in addition to low nuclear RBM3 expression was positively associated with tumor aggressiveness and UBC patients' poor outcomes. So our results suggest that these markers together are useful as predictive markers for progression and dismal outcome in patients with bladder cancer.

We recommend large studies to assess mechanisms of action of those markers in UBC.

The authors declare no conflict of interest. 


\section{References}

1. Yu D, Zhang H, Gui J. RNA-binding protein HuR promotes bladder cancer progression by competitively binding to the long noncoding HOTAIRwith miR-1. Onco Targets Ther 2017;10: 2609-2619.

2. Bambury RM, Rosenberg JE. Actionable mutations in muscle-invasive bladder cancer. Curr Opin Urol 2013; 23: 472-478.

3. Boman K, Andersson G, Wennersten Ch, Nodin B, Ahlgren G, Jirström K. Podocalyxin-like and RNA-binding motif protein 3 are prognostic biomarkers in urothelial bladder cancer: a validatory study. Biomark Res 2017; 5:10.

4. Babjuk M, Bohle A, Burger M, et al. EAU guidelines on non-muscle-invasive urothelial carcinoma of the bladder: Update 2016. Eur Urol 2017; 71: 447-461.

5. Sternberg CN, Bellmunt J, Sonpavde G, et al. ICUD-EAU International Consultation on bladder cancer 2012: chemotherapy for urothelial carcinoma-neoadjuvant and adjuvant settings. Eur Urol 2013; 63: 58-66.

6. Breau RH, Karnes RJ, Farmer SA, et al. Progression to detrusor muscle invasion during urothelial carcinoma surveillance is associated with poor prognosis. BJU Int 2014; 113: 900-906.

7. Kim do Y, Park EY, Chang E, et al. A novel miR-34a target, protein kinase D1, stimulates cancer stemness and drug resistance through GSK3/beta-catenin signaling in breast cancer. Oncotarget 2016; 7: 14791-14802.

8. Kishore S, Luber S, Zavolan M. Deciphering the role of RNA-binding proteins in the post-transcriptional control of gene expression. Brief Funct Genomics 2010; 9: 391-404.

9. Miyata Y, Watanabe S, Sagara Y, et al. High expression of HuR in cytoplasm, but not nuclei, is associated with malignant aggressiveness and prognosis in bladder cancer. PLoS One 2013; 8: e59095.

10. Ye F, Peisheng J, Xiaoniao C, PeiPei CH, Huimin C. An high RNA-bind ing motif protein 3 (RBM3) expression is independently associated with prolonged overall survival in intestinal-type gastric cancer. Med Sci Monit 2017; 23: 6033-6041.

11. Zhou RB, Xiao-Li L, Chen-Yan Z, Da-Chuan Y. RNA binding motif protein 3: a potential biomarker in cancer and therapeutic target in neuroprotection. Oncotarget 2017; 8: 22235-22250.

12. Nielsen JS, Graves ML, Chelliah S, Vogl AW, Roskelley CD, McNagny KM. The CD34-related molecule podocalyxin is a potent inducer of microvillus formation. PLoS One 2007; 2: e237.

13. Takeda T, Go WY, Orlando RA, Farquhar MG. Expression of podoca lyxin inhibits cell-cell adhesion and modifies junctional properties in Madin-Darby canine kidney cells. Mol Biol Cell 2000; 11: 3219-3232.

14. Nielsen JS, McNagny KM. Novel functions of the CD34 family. J Cell Sci 2008; 121: 3683-3692.

15. Kusumoto H, Shintani Y, Kanzaki R, et al. Podocalyxin influences malignant potential by controlling epithelial-mesenchymal transition in lung adenocarcinoma. Cancer Sci 2017; 108: 528-535.

16. Sobin LH, Wittekind C. TNM Classification of Malignant Tumours. Wiley-Liss, New York 2002.

17. Eble JN, Sauter G, Epstein JI, Sesterhenn IA. World Health Organization Classification of Tumours: Pathology and Genetics of Tumours of the Urinary System and Male Genital Organs. ARC Press, Lyon 2004.

18. Boman K, Segersten U, Ahlgren G, et al. Decreased expression of RNA-binding motif protein 3 correlates with tumour progression and poor prognosis in urothelial bladder cancer. BMC Urol 2013; 13: 17.

19. Sievert KD, Amend B, Nagele U, et al. Economic aspects of bladder cancer: what are the benefits and costs? World J Urol 2009; 27: 295-300.

20. Dong R, Yang GD, Luo NA, Qu YQ. HuR: a promising therapeutic target for angiogenesis. Gland Surg 2014; 3: 203-206.

21. Brennan CM, Steitz JA. HuR and mRNA stability. Cell Mil Life Sci 2011; 58: 341-347.

22. Wang J, Guo Y, Chu H, Guan, Bi J, Wang B. Multiple functions of the RNA-binding protein HuR in cancer progression, treatment responses and prognosis. Int J Mol Sci 2013; 14: 10015-10041.

23. Kim I, Kwak H, Lee HK, Hyun S, Jeong S. $\beta$-catenin recognized a specific RNA motif in the cyclooxygenase-2 mRNA \#'-UTR and interacts with HuR in colon cancer cells. Nucleic Acids Res 2012; 40: 6863-6872.
24. Yuan Z, Sanders AJ, Ye L, Wang Y, Jiang WG. Prognostic value of human antigen $\mathrm{R}(\mathrm{HuR})$ in human breast cancer: high level predicts a favourable prognosis. Anticancer Res 2011; 31: 303-310.

25. Srikantan S, Gorospe M. HuR function in disease. Front Biosci 2012; 17: 189- 205.

26. Sakuma T, Nakagawa T, Ido K, et al. Expression of vascular endothelial growth factor-A and mRNA stability factor HuR in human meningiomas. J Neurooncol 2008; 88: 143-155.

27. Ronkainen H, Vaarala MH, Hirvikoski P, Ristimaki A. HuR expression is a marker of poor prognosis in renal cell carcinoma. Tumour Biol 2011; 32: 481-487.

28. Florianova L, Xu B, Traboulsi S, et al. Evaluation of RNA-binding motif protein 3 expression in urothelial carcinoma of the bladder: an immunohistochemical study. World J Surg Oncol 2015; 13: 317.

29. Jang HH, Lee HN, Kim SY, et al. Expression of RNA-binding motif protein 3 (RBM3) and cold-inducible RNA-binding protein (CIRP) is associated with improved clinical outcome in patients with colon cancer. Anticancer Res 2017; 37: 1779-1785.

30. Olofsson SE, Nodin B, Gaber A, et al. Low RBM3 protein expression correlates with clinical stage, prognostic classification and increased risk of treatment failure in testicular non-seminomatous germ cell cancer. PLoS One 2015; 10: e0121300.

31. Ehlén A, Nodin B, Rexhepaj E, et al. RBM3-regulated genes promote DNA integrity and affect clinical outcome in epithelial ovarian cancer. Transl Oncol 2011; 4: 212-221.

32. Grupp K, Wilking J, Prien K, et al. High RNA-binding motif protein 3 expression is an independent prognostic marker in operated prostate cancer and tightly linked to ERG activation and PTEN deletions. Eur J Cancer 2014; 50: 852-861.

33. Fodde $\mathrm{R}$, Brabletz T. Wnt/ $\beta$-catenin signaling in cancer stemness and malignant behavior. Curr Opin Cell Biol 2007; 19: 150-158.

34. Venugopal A, Subramaniam D, Balmaceda J, et al. RNA binding protein RBM3 increases beta-catenin signaling to increase stem cell characteristics in colorectal cancer cells. Mol Carcinog 2016; 55: 1503-1516.

35. Sureban SM, Ramalingam S, Natarajan G, et al. Translation regulatory factor RBM3 is a proto-oncogene that prevents mitotic catastrophe. Oncogene 2008; 27: 4544-4556.

36. Wellmann S, Bührer C, Moderegger E, et al. Oxygen-regulated expression of the RNA-binding proteins RBM3 and CIRP by a HIF-1-independent mechanism. J Cell Sci 2004; 117: 1785-1794.

37. Kusumoto H, Shintani Y, Kanzaki R, et al. Podocalyxin influences malignant potential by controlling epithelial-mesenchymal transition in lung adenocarcinoma. Cancer Sci 2017; 108: 528-535.

38. Meng X, Ezzati P, Wilkins JA. Requirement of podocalyxin in TGF-beta induced epithelial mesenchymal transition. PLoS One 2011; 6: e18715.

39. Larsson AH, Nodin B, Syk I, et al. Podocalyxin-like protein expression in primary colorectal cancer and synchronous lymph node metastases. Diagn Pathol 2013; 8: 109.

40. Wu H, Yang L, Liao D, Chen Y, Wang W, Fang J. Podocalyxin regulates astrocytoma cell invasion and survival against temozolomide. Exp Ther Med 2013; 5: 1025-1029.

41. Somasiri A, Nielsen JS, Makretsov N, et al. Overexpression of the anti-adhesin podocalyxin is an independent predictor of breast cancer progression. Cancer Res 2004; 64: 5068-5073.

42. Poletajew S, Braticevici B, Brisuda A, et al. Timing of radical cystectomy in Central Europe - multicenter study on factors influencing the time from diagnosis to radical treatment of bladder cancer patients. Cent European J Urol 2015; 68: 9-14.

\section{Address for correspondence}

\section{Walid S.H. Elsayed, MD}

Pathology Faculty of Medicine

Zagazig University

Zagazig, Egypt

e-mail: walid_shaban@gmail.com

Submitted: 28.10 .2021

Accepted: 23.11.2021 